\title{
Análise estrutural e considerações sobre a dinâmica sucessional de dois fragmentos florestais semideciduais do Jardim Botânico Municipal de Bauru, SP, Brasil ${ }^{1}$
}

\author{
Marcelo Henrique Ongaro Pinheiro ${ }^{2,4}$ e Reinaldo Monteiro ${ }^{3}$
}

Recebido em 10/11/2008. Aceito em 21/01/2009

\begin{abstract}
RESUMO - (Análise estrutural e considerações sobre a dinâmica sucessional de dois fragmentos florestais semideciduais do Jardim Botânico Municipal de Bauru, SP, Brasil). Parâmetros fitossociológicos, como freqüência de classes de altura e de diâmetro, são muito utilizados em inferências sobre sucessão secundária em fitocenoses. Indivíduos abustivo-arbóreos com no mínimo $1,5 \mathrm{~m}$ de altura e fuste a $1,3 \mathrm{~m}$, foram amostrados em $2.600 \mathrm{~m}^{2} \mathrm{de}$ floresta estacional semidecidual do Jardim Botânico Municipal de Bauru (JBMB). Informações sobre as freqüências de classes de diâmetro das cinco espécies com maior índice de valor de importância (Ocotea pulchella, Protium heptaphyllum, Copaifera langsdorffii, Platypodium elegans e Trichilia pallida) e a classificação sucessional das populações mais numerosas também foram analisadas para inferências sobre as características sucessionais da comunidade estudada. As freqüências de classes de diâmetro de Vochysia tucanorum, espécie típica de savana, também foi estudada. As informações obtidas permitiram considerar que a floresta do JBMB encontra-se em estádio sucessional intermediário, e que, embora tenha sofrido com a ação do fogo no passado, algumas dessas populações estão conseguindo renovar-se.
\end{abstract}

Palavras-chave: ecótono, fogo, microambiente, sucessão secundária

ABSTRACT - (Structural analysis and considerations on the successional dynamic of two semideciduous forest fragments at the Municipal Botanical Garden of Bauru, SP, Brazil). Phytosociological parameters, as the frequency of height and diameter classes, are commonly used to make inferences about secondary succession in given phytocenoses. Shrub and tree individuals higher than $1.5 \mathrm{~m}$ and with a bole length of at least $1.3 \mathrm{~m}$ were sampled in 2,600 $\mathrm{m} 2$ of semideciduous seasonal forest in the Municipal Botanical Garden of Bauru (JBMB). Information on the diameter class frequencies of five species with the highest Importance Value Index (Ocotea pulchella, Protium heptaphyllum, Copaifera langsdorffii, Platypodium elegans and Trichilia pallida) and the more numerous species' successional classification were analyzed to make inferences about the successional features of the studied community. The diameter class frequencies of savannic species Vochysia tucanorum were also studied. The information obtained allowed to consider that the forest of the JBMB is at an intermediate successional stage, and that, although it used to be periodically burnt, some of its populations are able to renew themselves.

Key words: ecotone, fire, microenvironment, secondary succession

\section{Introdução}

A fragmentação de uma matriz florestal causa isolamento dos remanescentes, além de culminar em alterações das características ambientais locais (Saunders et al. 1991). O efeito de borda também é um outro fenômeno prejudicial ao remanescente ou fragmento (Tabanez et al. 1997), por contribuir para alterações de fatores abióticos e bióticos característicos de uma floresta (Longman \& Jeník 1992), afetando sua sustentabilidade (Viana \& Tabanez 1996). Essas mudanças influenciam o desenvolvimento do chamado ciclo mosaico, conceito cunhado por Aubreville (Remmert 1991), ou da sucessão vegetal, que foi descrita por elaborada nomenclatura desenvolvida por Clements (Barnes et al. 1997) que, por sua vez, foi influenciado pelos estudos de Thoreau e Cowles (Whitmore 1982; Barnes et al. 1997).

Para Whitmore (1982), as formações florestais apresentariam similaridade quanto aos mecanismos relacionados à sucessão, e as etapas clareira, desenvolvimento e madura poderiam ser arbitrariamente distinguidas. Haveria a participação de diásporos de diferentes espécies vegetais participando desse mecanismo, que seriam transportados para o local perturbado (Schupp et al. 1988; Mueller-Dombois 1995; Barnes et al. 1997) por diferentes vetores (Schupp et al. 1988). Desta maneira, espécies não florestais podem ser beneficiadas pelo surgimento de clareiras, através do trans- porte de suas sementes por animais centenas de metros para o interior da floresta, atingindo áreas perturbadas (Terborgh 1992). Todavia, o vento pode conduzir sementes para clareiras com mais eficiência que a própria zoocoria (Schupp et al. 1988). Ao longo do desenvolvimento do mecanismo sucessional em uma floresta semidecidual, o aumento de cobertura vegetal ocorreria em detrimento de espécies intolerantes à diminuição da radiação solar (Oliveira-Filho et al. 1997).

As dimensões de uma clareira recém criada em uma formação florestal seriam decisivas na definição da composição das espécies vegetais que a ocupariam, entre espécies tolerantes e intolerantes à luminosidade (Brokaw 1985a; Brokaw \& Scheiner 1989), a partir de estratégias regenerativas distintas (Grime \& Hillier 1993). A variação da intensidade luminosa, portanto, seria um importante fator para a definição de nichos para espécies vegetais (Newman 1982), mesmo ao longo do gradiente estabelecido pela variação da luminosidade na transição campina/floresta (Martens et al. 2000).

A freqüência de classes de diâmetro é um critério para definir estágios de desenvolvimento de uma floresta (Martins 1991), auxiliando avaliações sobre o desenvolvimento florestal (Condit et al. 1998). É um descritor utilizado por muitos autores em diferentes análises (Hatfield et al. 1996; Mesquita et al. 2001), como a caracterização estrutural de diferentes formações florestais (Lopes et al. 2002b; Gomes

\footnotetext{
Parte da dissertação de Mestrado do primeiro Autor

Universidade Federal de Uberlândia (UFU), Faculdade de Ciências Integradas do Pontal (FACIP), Ituiutaba, MG, Brasil

Universidade Estadual Paulista (UNESP), campus de Rio Claro, Rio Claro, SP, Brasil

Autor para correspondência: mhop@pontal.ufu.br
} 
et al. 2005), subsídio às práticas de conservação e manejo (Peixoto et al. 2005; Souza et al. 2006), além de possibilitar inferências sobre a ocorrência de perturbações pretéritas (Martins 1991; Gomes et al. 2005), a dinâmica de populações mais freqüentes e características sucessionais (Martins 1991). Estimativas das alturas dos indivíduos arbustivoarbóreos podem fornecer informações importantes tanto para a interpretação de estrutura vertical da floresta como auxiliar na compreensão da dinâmica das populações que a compõem (Martins 1991).

O presente estudo traz informações das características estruturais de uma floresta estacional semidecidual, fornecendo hipóteses para fenômenos relacionados à sucessão secundária de florestas estacionais similares à encontrada no Jardim Botânico Municipal de Bauru. Foram realizadas inferências sobre a contribuição de espécies florestais e savânicas na dinâmica regenerativa na área de estudo.

\section{Material e métodos}

Área de estudo - O presente trabalho foi desenvolvido em dois fragmentos de floresta estacional semidecidual (5 e $7 \mathrm{ha}$ ) contíguos, localizados no Jardim Botânico Municipal de Bauru (JBMB), estado de São Paulo. As coordenadas $22^{\circ} 20^{\prime} 39^{\prime}$ ' S e $49^{\circ} 00^{\prime} 51^{\prime}$ 'W fornecem a localização aproximada do centro do fragmento florestal menor. Além dessa fitocenose, o JBMB abriga também um grande remanescente de cerradão (277 ha), ou savana florestada (Veloso 1992), e um pequeno fragmento (1 ha) de mata de brejo (Rodrigues 2000), junto do córrego Vargem Limpa. A savana florestada ocupa locais mais elevados no terreno, e entra em contato, em cotas topográficas menores, com a floresta estacional semidecidual (Pinheiro et al. 2002; Pinheiro \& Monteiro 2006).

Os dois fragmentos florestais, no passado, abrangiam uma área maior, mas, ao longo dos anos, foram reduzidos aos limites atuais. Em ambos, mesmo em locais distantes da borda, foram encontrados por Pinheiro et al. (2002) muitos indivíduos arbóreos com a superfície do ritidoma queimada, um sinal evidente da ocorrência de fogo. O histórico da reserva mantida pelo JBMB registra incêndios periódicos, normalmente coincidindo com a estiagem. Em 1994, por exemplo, ano de prolongada seca e geada invernais, foi registrado um grande incêndio. Outra indicação de perturbações pretéritas foi a observação da presença de grande número de lianas nos fragmentos florestais. Em alguns locais formavam, junto ao solo e ao redor de árvores, um emaranhado denso. Vale ressaltar que a ocorrência de clareiras favorece o desenvolvimento de lianas (Bazzaz \& Pickett 1980). Pôde ser encontrada ainda a espécie Pteridium af. aquilinum (L.) Kuhn, comum em bordas de mata e em locais perturbados (Lorenzi 2000); Heliconia psittacorum L.f., definida como heliófita por Lorenzi \& Souza (2001), além de espécies citadas como indicadoras da ocorrência de clareiras em ambientes florestais, como Cordia sellowiana Cham., Pera glabrata (Schott) Poepp. ex Baill., Croton floribundus Spreng. (Tabarelli \& Mantovani 1997a; 1997b) e Trema micrantha (L.) Blume (Brokaw 1989). Essas espécies também foram coletadas na savana florestada contígua à floresta estudada por Pinheiro et al. (2002) e Pinheiro \& Monteiro (2008). No interior da floresta estacional, especialmente no fragmento menor, foram observadas touceiras de Heliconia pouco desenvolvidas, por influência provável da menor incidência de luz, conseqüência da regeneração local (Pickett \& White 1986). Fora dos fragmentos florestais, em áreas destituídas de qualquer cobertura vegetal arbórea, foi possível observar $H$. psittacorum formando touceiras densas. No interior dos fragmentos, também foram encontradas Cecropia catarinensis Cuatrec. e Cecropia cf. lyratiloba Miq. O gênero Cecropia Loefl. foi definido por Brokaw (1985a; 1985b) como característico de grandes clareiras. Ainda sobre o histórico da área de estudo, relatos de antigos servidores municipais atestam que, muito antes da criação do JBMB, eventualmente a floresta estacional fornecia toras para finalidades diversas, como para a construção de pontes.

Metodologia - A análise estrutural baseou-se em levantamento fitossociológico desenvolvido em 26 parcelas, de $10 \mathrm{~m} \times 10 \mathrm{~m}$ cada uma, totalizando $2.600 \mathrm{~m}^{2}$ tendo sido evitada a proximidade de trilhas e de bordas. Do montante de parcelas, 18 foram instaladas no fragmento de cinco hectares, divididas em três blocos com seis parcelas cada um, posicionados de maneira que a distância mínima entre blocos fosse de $40 \mathrm{~m}$. No fragmento de sete hectares, optou-se pela delimitação de um único bloco, constituído por oito parcelas, no centro do fragmento. Desta forma, tentou-se evitar a proximidade das bordas, muito afetadas por incêndios freqüentes, e de trilhas. Gráficos contendo as curvas do coletor ou curva de espécies e área (Braun-Blanquet 1979) foram produzidos separadamente para os dois fragmentos.

Informações sobre as características fitossociológicas e florísticas da área estudada poderão ser encontradas em Pinheiro et al. (2002) e Pinheiro \& Monteiro $(2006 ; 2008)$. No presente estudo, foram incluídos todos os indivíduos com no mínimo 1,5 m de altura e fuste a 1,3 m do solo. Segundo Martins (1991), a inclusão das árvores de altura igual ou superior ao estrato mais baixo, com as medições tomadas à altura do peito, i.e. $1,3 \mathrm{~m}$, oferece aos polígonos de freqüência das classes de diâmetro maior consistência. Desta forma, as medições possibilitaram que as menores árvores fossem consideradas na amostragem.

Os histogramas de freqüência de classes de diâmetro foram elaborados de acordo com a metodologia encontrada em Spiegel (1976). Histogramas para classes de diâmetro foram elaborados para as cinco espécies com os maiores índices de valor de importância (IVI), apresentadas por Pinheiro et al. (2002), Ocotea pulchella (Nees) Mez, Protium heptaphyllum (Aubl.) Marchand, Copaifera langsdorffii Desf., Platypodium elegans Vogel, Trichilia pallida Sw. Além disso, as variações diamétricas de Vochysia tucanorum Mart., espécie savânica, amostrada no interior da floresta estacional estudada (Pinheiro \& Monteiro 2008), também foram analisadas. As espécies relacionadas acima representaram exemplos dos padrões de distribuição das classes de freqüência de diâmetro encontrados na área de estudo. Foi elaborada também, a análise sobre freqüências de classes de altura da comunidade florestal estudada, isto é, do conjunto das medidas verticais de todos os indivíduos amostrados.

Foi realizada também análise das classes sucessionais das espécies encontradas na floresta do JBMB, e estudadas por Pinheiro et al. (2002) e Pinheiro \& Monteiro (2008). Para tanto, as classificações sucessionais das espécies, como pioneiras, secundárias iniciais e secundárias tardias, basearam-se nos trabalhos de Durigan \& Nogueira (1990), Durigan \& Leitão Filho (1995), Gandolfi et al. (1995), Lorenzi (1992; 2002), Lopes et al. (2002a), Stranghetti et al. (2003) e Marangon et al. (2007). Para as espécies não mencionadas por esses autores, observações de campo auxiliaram na definição das respectivas categorias sucessionais. $\mathrm{O}$ trabalho de Pinheiro \& Monteiro (2006), por sua vez, foi utilizado para a caracterização de espécies como savânicas, isto é, quando formações savânicas representassem o habitat de origem dessas espécies. O número total de indivíduos, alturas mínima, média e máxima, para cada uma das espécies, também foram considerados.

\section{Resultados e discussão}

A partir da análise das classes sucessionais das espécies e das populações com no mínimo 10 indivíduos, foi possível definir o estádio sucessional da floresta estacional do JBMB como intermediário. Dos indivíduos amostrados das populações com no mínimo 10 indivíduos, excluindo-se os mortos, isto é, $86 \%$ do total dos indivíduos encontrados no 0,26 ha estudado por Pinheiro et al. (2002), área amostral considerada suficiente (Fig. 1), 4,3\% foram classificados como pioneiros, $29,1 \%$ como secundários iniciais, $36,1 \%$ como secundários tardios, e 16,3\% não puderam ser classificados. Quanto às 192 espécies encontradas por Pinheiro \& Monteiro (2008) no mesmo fragmento, 77 não puderam ser incluídas em nenhuma das três classes sucessionais acima. Contudo, 30 espécies foram definidas como pioneiras, 41 como secundárias iniciais, e as secundárias tardias totaliza- 
ram 44 espécies. Entre as espécies mais numerosas, O. pulchella, C. langsdorffii, P. elegans, foram classificadas como secundárias iniciais, e P. heptaphyllum e T. pallida, como secundárias tardias (Tab. 1). Essas cinco espécies foram as mais importantes quanto ao IVI, no estudo realizado por Pinheiro et al. (2002). Para Lopes et al. (2002b) a ocorrência concomitante de espécies, com exigências ambientais distintas entre as mais destacadas, é possível quando há a exploração de recursos ambientais distintos em uma floresta.

O padrão de distribuição de classes de alturas e de diâmetros, observado no presente estudo, foi similar ao registrado em outras formações florestais, como as descritas por Lopes et al. (2002b) no Parque Estadual do Rio Doce (MG), Nascimento et al. (2004) em Monte Alegre (GO), Peixoto et al. (2005) no Rio de Janeiro (RJ), e Souza et al. (2006) em Paragominas (PA). Como nas fitocenoses florestais dessas localidades, o elevado número de indivíduos, nas duas ou três menores classes diamétricas e de altura da floresta do JBMB (Fig. 2 e 3), é um importante indicativo sobre a capacidade de renovação da floresta do JBMB, pelo estabelecimento de indivíduos arbóreos de pequeno porte no chamado estrato regenerativo (Pinto et al. 2005). Entretanto, o critério de inclusão utilizado no presente estudo, contribuiu para a elevada concentração de indivíduos nas menores classes de diâmetro, especialmente na primeira, que deteve $76,2 \%$ do total de indivíduos amostrados. Foi também responsável pelas curvas acentuadas com forma de "J" invertido, nas distribuições de freqüências de classes de altura como de diâmetro.

A ausência de indivíduos em três das maiores classes de diâmetro, nos intervalos correspondentes a 54-58,5; 63-67,5 e 67,5-72 cm (Fig. 3), pode estar relacionada à ocorrência de perturbações pretéritas. Situação similar foi encontrada por Lopes et al. (2002b), em Minas Gerais, onde os autores inferiram que a ausência de indivíduos em uma classe de diâmetro, e a presença de indivíduos em classe imediatamente acima no histograma de distribuição de freqüências de classes, representaria a sobrevivência de árvores a incêndios ocorridos na área.

Três espécies, entre as mais importantes para o IVI (Pinheiro et al. 2002), O. pulchella, C. langsdorffii e $P$. elegans (Fig. 4 e 5), mostraram distribuição de classes de diâmetro truncada, assim como V. tucanorum (Fig. 6). Para Gomes et al. (2005) esse tipo de distribuição, definido por um número menor de indivíduos em uma classe, em relação ao intervalo seguinte pode ser associado a períodos anteriores de perturbação. Todavia, pode-se afirmar que essas espécies, com exceção de $V$. tucanorum, apresentaram um recrutamento contínuo, com diminuição progressiva do número de indivíduos, da menor para as maiores classes de diâmetro, padrão similar às distribuições das classes de diâmetro de $O$. pulchella, P. heptaphyllum e C. langsdorffii (Fig. 4 e 5). Não obstante, todas as espécies acima apresentaram ausência de indivíduos em pelo menos duas classes diamétricas, o que pode representar períodos de insucesso para o estabelecimento de suas plântulas. Oliveira-Filho et al. (1996) consideraram C. langsdorffii como uma espécie ambígua, sendo abundante tanto na mata como no cerrado. A ocorrência de indivíduos dessa espécie ocupando as menores classes de diâmetro confirma sua capacidade de estabelecerse e desenvolver-se no sub-bosque florestal (Oliveira-Filho et

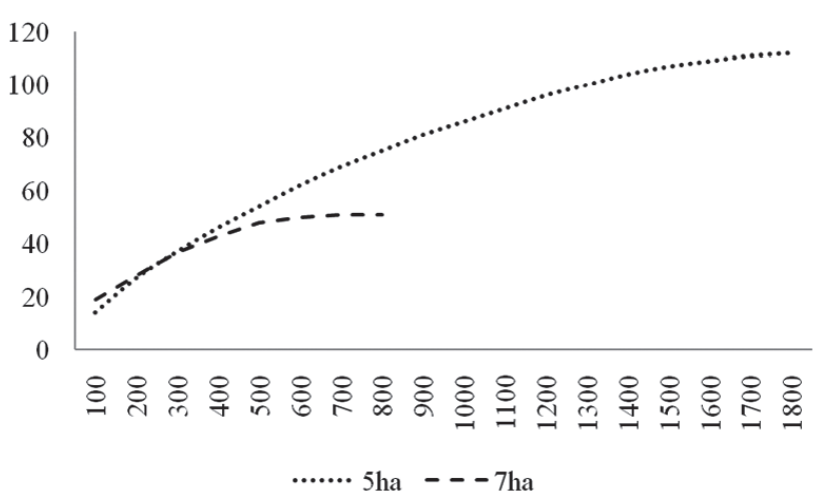

Figura 1. Curvas do coletor (número de espécies - ordenada; área amostral abscissa) produzidas a partir dos blocos de parcelas instalados nos fragmentos menor (5 ha) e maior (7 ha).

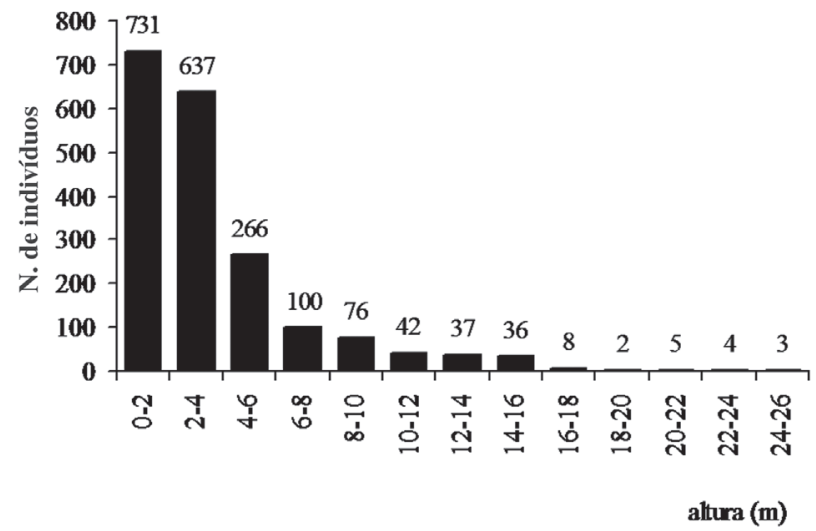

Figura 2. Distribuição das classes de altura dos indivíduos arbustivo-arbóreos amostrados na floresta estacional semidecidual do Jardim Botânico Municipal de Bauru, SP, Brasil. Esse histograma foi definido com intervalos de classe igual a $2,0 \mathrm{~m}$.

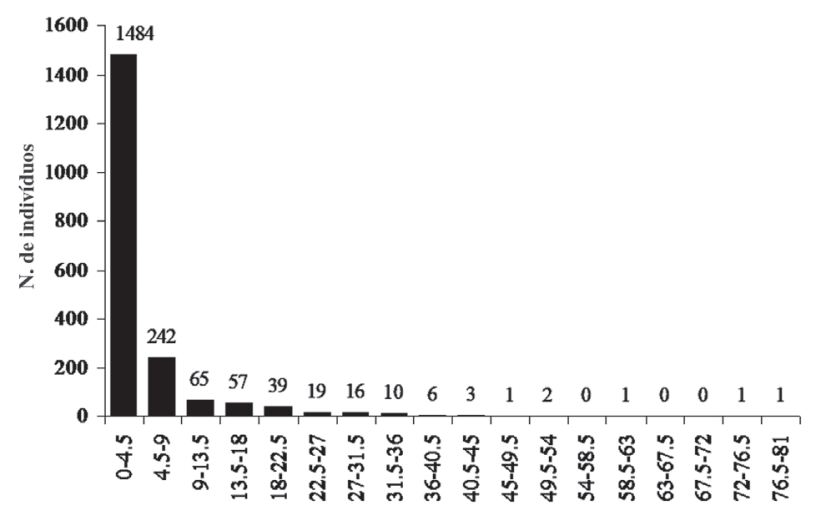

dỉimetro (cm)

Figura 3. Distribuição das classes de diâmetro dos indivíduos arbustivo-arbóreos amostrados na floresta estacional semidecidual do Jardim Botânico Municipal de Bauru, SP, Brasil. Esse histograma foi definido com intervalos de classe igual a $4,5 \mathrm{~m}$. 
al. 1996). E, sendo uma espécie generalista (Oliveira-Filho \& Ratter 2002), podendo ser encontrada em formações florestais semideciduais, ciliares e savânicas, como mencionaram Lobo \& Joly (2000), a ausência de indivíduos em três das classes de diâmetro, corrobora a hipótese de perturbações na floresta do JBMB.

Por sua vez, V. tucanorum, definida como savânica por Pinheiro \& Monteiro (2006), necessitaria de certa disponibilidade de luz para o estabelecimento de suas populações, como a luminosidade encontrada em transições savânicoflorestais (Oliveira-Filho \& Ratter 2002). Seria possível, portanto, a presunção de que a ausência de indivíduos de $V$. tucanorum em nove, das 14 classes de diâmetro (Fig. 6), teria ocorrido por diminuição progressiva da incidência luminosa pelo aumento da cobertura vegetal florestal, mesmo em períodos pré-climácicos. Assim, como mencionaram Barbosa et al. (1999), ambientes com níveis intermediários de luz, como os encontrados em pequenas clareiras, favoreceriam a germinação de sementes de $V$. tucanorum, ao contrário da baixa incidência luminosa. A não ocorrência de indivíduos menores que 2,0 $\mathrm{m}$ de altura (Tab. 1) contribuiu para a proposição acima. Entretanto, a ação de incêndios, como fator determinante para a ausência de indivíduos $V$. tucanorum em muitas classes de altura, não deve ser descartada. A respeito da influência do fogo sobre $V$. tucanorum, espécie comum em savanas florestadas (Durigan et al. 2004), e abundante na transição com as demais fisionomias savânicas, incêndios freqüentes diminuiriam a densidade de suas populações, favorecendo o estabelecimento de fisionomias com predomínio de herbáceas (Oliveira-Filho \& Ratter 2002).

O maior número de indivíduos de $C$. vernalis, M. elaeagnoides e $X$. aromatica, espécies definidas como iniciais em uma sucessão, em classes menores de distribuição diamétrica, é uma indicação de que passaram a se estabelecer com maior eficiência na floresta estacional do JBMB há pouco tempo. A ocorrência de diferentes microhabitats originados por perturbações (Mueller-Dumbois 1995), como clareiras (Whitmore 1989; Brokaw \& Scheiner 1989), ou a proximidade da borda em um fragmento (Nunes et al. 2003; Rodrigues et al. 2003), locais com maior luminosidade (Remmert 1991; Terborgh et al. 1997), podem ter fornecido as condições necessárias para a ocupação dessas espécies.

A presença de espécies, definidas por Leitão Filho (1992) e Pinheiro \& Monteiro (2006) como comuns ou típicas de formações savânicas, com populações compostas por no máximo seis indivíduos, mas com alturas médias elevadas, como Gochnatia polymorpha (Less.) Cabrera, Qualea cordata Spreng. e Qualea grandiflora Mart. (Tab. 2), corrobora a hipótese de troca de elementos florísticos entre as duas fitocenoses, fenômeno discutido por Pinheiro \& Monteiro (2006; 2008), indicando a participação da savana florestada contígua no mecanismo de sucessão na floresta estacional. Os diásporos de espécies savânicas passariam a ocupar a floresta estacional em locais com características microambientais temporariamente adequadas às espécies savânicas (Pinheiro \& Monteiro 2008), todavia,
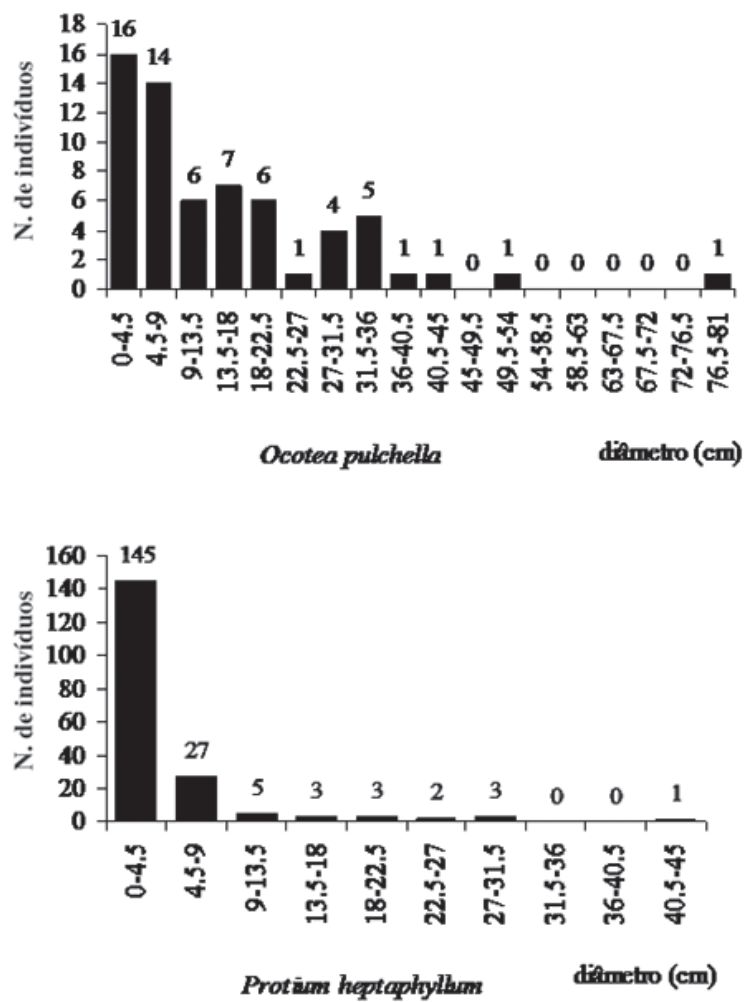

Figura 4. Distribuição das classes de diâmetro dos indivíduos pertencentes à Ocotea pulchella e Protium heptaphyllum amostrados na floresta estacional semidecidual do Jardim Botânico Municipal de Bauru, SP, Brasil.
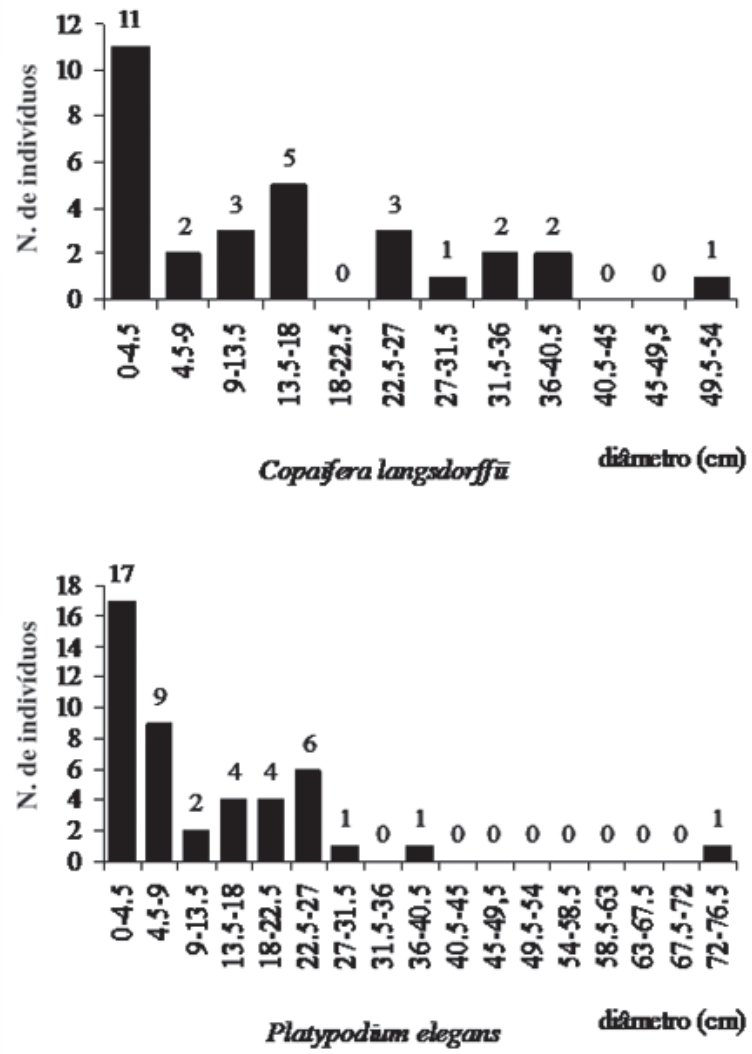

Figura 5. Distribuição das classes de diâmetro dos indivíduos pertencentes à Copaifera langsdorffi e Platypodium elegans amostrados na floresta estacional semidecidual do Jardim Botânico Municipal de Bauru, SP, Brasil. 
Tabela 1. Classificação sucessional, número de indivíduos e valores para altura das espécies com no mínimo 10 indivíduos, amostradas na floresta estacional do Jardim Botânico de Bauru. Ni - número de indivíduos amostrados por Pinheiro et al. (2002). CS - classes sucessionais. Alturas: mínimas (Min.); médias (Med.); máximas (Max.). Pi - pioneiras. Si - secundária inicial. St - secundária tardia. n - espécie não definida quanto ao estádio sucessional.

\begin{tabular}{|c|c|c|c|c|c|}
\hline \multirow{2}{*}{ Espécies } & \multirow{2}{*}{$\mathrm{Ni}$} & \multirow{2}{*}{$\mathrm{CS}$} & \multicolumn{3}{|c|}{ Alturas (m) } \\
\hline & & & Min. & Med. & Max. \\
\hline Xylopia aromatica (Lam.) Mart. & 22 & $\mathrm{Pi}$ & 1,5 & 2,5 & 8,0 \\
\hline Aspidosperma cylindrocarpon Müll. Arg. & 21 & St & 1,5 & 8,0 & 22,0 \\
\hline Dendropanax cuneatus (DC.) Decne. \& Planch. & 20 & St & 1,5 & 2,6 & 7,0 \\
\hline Zeyheria tuberculosa (Vell.) Bureau & 13 & $\mathrm{Pi}$ & 2,0 & 7,0 & 16,0 \\
\hline Protium heptaphyllum (Aubl.) Marchand & 189 & St & 1,5 & 4,3 & 17,0 \\
\hline Copaifera langsdorffii Desf. & 30 & $\mathrm{Si}$ & 1,5 & 10,3 & 25,0 \\
\hline Erythroxylum subracemosum Turcz. & 24 & $\mathrm{n}$ & 1,5 & 2,1 & 5,0 \\
\hline Croton floribundus Spreng. & 15 & $\mathrm{Pi}$ & 1,5 & 6,8 & 15,0 \\
\hline Machaerium brasiliense Vogel & 10 & St & 1,5 & 3,0 & 5,0 \\
\hline Machaerium hirtum (Vell.) Stellfeld & 20 & $\mathrm{Si}$ & 1,5 & 3,6 & 8,0 \\
\hline Platypodium elegans Vogel & 45 & $\mathrm{Si}$ & 1,5 & 8,3 & 25,0 \\
\hline Sweetia fruticosa Spreng. & 19 & St & 2,0 & 4,2 & 9,0 \\
\hline Lacistema hasslerianum Chodat & 37 & $\mathrm{n}$ & 1,5 & 2,3 & 14,0 \\
\hline Endlicheria paniculata (Spreng.) J.F. Macbr. & 19 & St & 1,5 & 7,8 & 14,0 \\
\hline Ocotea pulchella (Nees) Mez & 63 & $\mathrm{Si}$ & 1,5 & 9,2 & 24,0 \\
\hline Trichilia pallida $\mathrm{Sw}$. & 166 & St & 1,5 & 2,9 & 10,0 \\
\hline Inga vera Willd. ssp. affinis & 18 & $\mathrm{Pi}$ & 1,5 & 4,6 & 10,0 \\
\hline Mollinedia widgrenii A. DC. & 35 & St & 1,5 & 4,4 & 11,0 \\
\hline Siparuna guianensis Aubl. & 52 & St & 1,5 & 2,5 & 4,0 \\
\hline Sorocea cf. bonplandii (Baill.) W.C. Burger, Lanj. \& Wess. Boer & 18 & $\mathrm{n}$ & 1,5 & 3,2 & 7,0 \\
\hline Rapanea umbellata (Mart.) Mez & 50 & St & 1,5 & 3,1 & 7,0 \\
\hline Stylogyne ambigua (Mart.) Mez & 40 & $\mathrm{n}$ & 1,5 & 2,4 & 6,0 \\
\hline Campomanesia guaviroba (DC.) Kiaersk. & 12 & St & 1,5 & 3,2 & 5,0 \\
\hline Campomanesia guazumifolia (Cambess.) O. Berg & 10 & St & 1,5 & 2,5 & 5,0 \\
\hline Eugenia florida DC. & 21 & St & 1,5 & 3,6 & 11,0 \\
\hline Myrcia tomentosa (Aubl.) DC. & 38 & $\mathrm{Si}$ & 1,5 & 5,7 & 16,0 \\
\hline Myrciaria ciliolata (Cambess.) O. Berg & 13 & $\mathrm{n}$ & 1,5 & 1,8 & 3,0 \\
\hline Roupala brasiliensis Klotzsch & 10 & St & 1,5 & 3,5 & 6,0 \\
\hline Alibertia macrophylla K. Schum. & 31 & St & 1,5 & 2,5 & 6,0 \\
\hline Chomelia obtusa Cham. \& Schltdl. & 20 & $\mathrm{n}$ & 1,5 & 3,3 & 9,0 \\
\hline Coussarea hydrangaefolia (Benth.) Benth. \& Hook. f. ex Müll. Arg. & 50 & $\mathrm{n}$ & 1,5 & 2,5 & 6,0 \\
\hline Cupania vernalis Cambess. & 140 & $\mathrm{Si}$ & 1,5 & 4,0 & 16,0 \\
\hline Cupania zanthoxyloides Cambess. & 77 & $\mathrm{n}$ & 1,5 & 3,5 & 10,0 \\
\hline Matayba elaeagnoides Radlk. & 143 & $\mathrm{Si}$ & 1,5 & 3,6 & 16,0 \\
\hline Chrysophyllum marginatum (Hook. \& Arn.) Radlk. & 10 & St & 1,5 & 4,7 & 10,0 \\
\hline Cestrum sp. & 21 & $\mathrm{n}$ & 1,5 & 1,9 & 3,0 \\
\hline Guazuma ulmifolia Lam. & 11 & $\mathrm{Pi}$ & 1,5 & 10,6 & 23,0 \\
\hline Luehea grandiflora Mart. & 30 & $\mathrm{Si}$ & 1,5 & 4,4 & 9,0 \\
\hline Vochysia tucanorum Mart. & 15 & $\mathrm{Si}$ & 2,0 & 8,9 & 15,0 \\
\hline
\end{tabular}

Tabela 2. Número de indivíduos e valores para altura de espécies savânicas, amostradas na floresta estacional do Jardim Botânico de Bauru, e classificadas como secundárias iniciais. Ni - número de indivíduos amostrados por Pinheiro et al. (2002). Alturas: mínimas (Min.); médias (Med.); máximas (Max.).

\begin{tabular}{|c|c|c|c|c|}
\hline \multirow{2}{*}{ Espécies } & \multirow{2}{*}{$\mathrm{Ni}$} & \multicolumn{3}{|c|}{ Alturas (m) } \\
\hline & & Min. & Med. & Max. \\
\hline Gochnatia polymorpha (Less.) Cabrera & 4 & 6,0 & 10,3 & 14,0 \\
\hline Qualea cordata (Mart.) Spreng. & 6 & 7,0 & 10,5 & 15,0 \\
\hline Qualea grandiflora Mart. & 4 & 4,0 & 8,5 & 12,0 \\
\hline
\end{tabular}


a regeneração da cobertura florestal conduziria o ambiente à condição original de baixa luminosidade. Foi observado que $G$. polymorpha e $Q$. cordata não apresentaram indivíduos na menor classe de altura (Tab. 2). Assim, deduz-se que essas espécies não estariam renovando suas populações, devido à competição direta com as espécies florestais. Tão logo fossem cessadas as perturbações, e com o início da sucessão secundária na fitocenose florestal, teria havido ocupação de espécies savânicas nos locais perturbados. Contudo, com o decorrer do processo regenerativo, essas espécies savânicas teriam sido substituídas, pouco a pouco, por espécies florestais, adaptadas ao ambiente de baixa luminosidade. Portanto, é possível supor que o fogo teve um papel fundamental na definição da composição florística atual, enriquecida por espécies savânicas. Ritidomas queimados foram freqüentes, mesmo em locais centrais da floresta, indicando a alta intensidade e, possivelmente, freqüência de incêndios na área de estudo.

As considerações de Pickett et al. (1987) de que perturbações, entre elas o fogo, podem favorecer a ocupação de espécies adaptadas ao microambiente criado, também corroboram a hipótese acima. Não obstante, a freqüência e a intensidade das perturbações são decisivas para o curso da sucessão secundária (Pickett et al. 1987), definindo a permanência ou a exclusão de populações (Higgins et al. 2000). Além disso, variações da porcentagem de colonização exercem importante influência sobre a composição florística no decorrer da sucessão (Bartha et al. 2003).
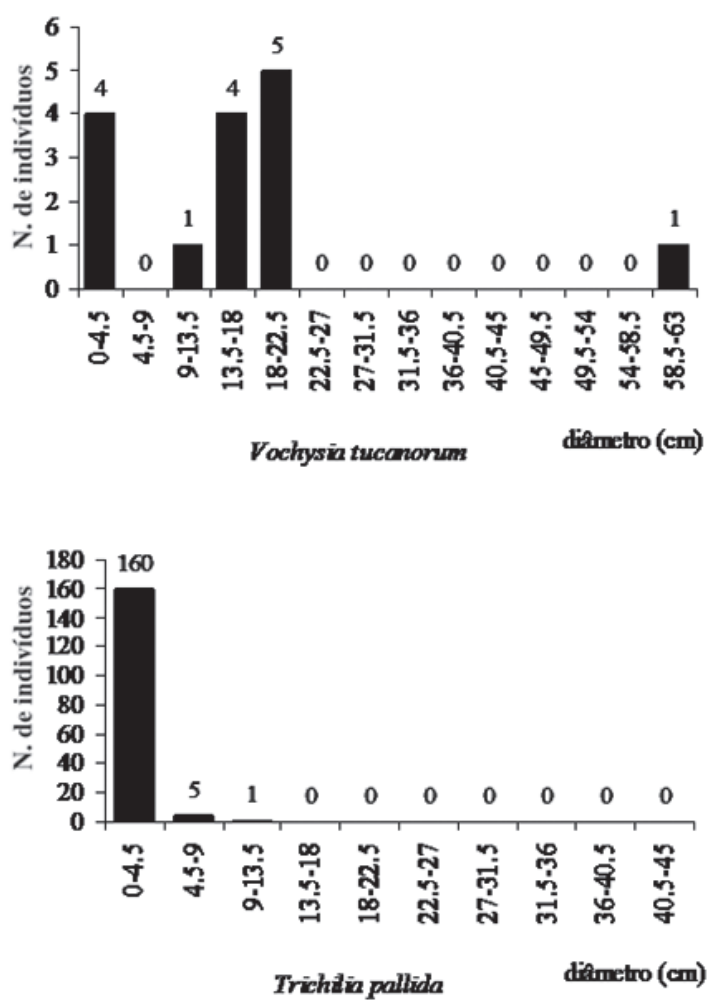

Figura 6. Distribuição das classes de diâmetro dos indivíduos pertencentes à Vochysia tucanorum e Trichilia pallida amostrados na floresta estacional semidecidual do Jardim Botânico Municipal de Bauru, SP, Brasil.
Histogramas de freqüência de classes de diâmetro com distribuições não balanceadas das classes de diâmetro, a partir da presença de interrupções em classes superiores, tanto para a comunidade florestal (Fig. 3) como para as populações analisadas, como $O$. pulchella, P. heptaphyllum, $C$. langsdorffi, P. elegans e V. tucanorum (Fig. 4, 5 e 6), pode também indicar a ocorrência de perturbações. E, embora o predomínio de indivíduos nas duas menores classes de diâmetro, $88,6 \%$ do total dos indivíduos amostrados para a comunidade, indique renovação da floresta do JBMB, três interrupções entre as classes de maior diâmetro permitem a inferência sobre perturbações pretéritas (Fig. 3). Essas espécies, além de possuírem alguns dos espécimes de maior porte, amostrados na área de estudo (Tab. 1), apresentaram um padrão similar de distribuição de classes de diâmetro, consistindo na ocorrência de indivíduos em pelo menos quatro classes de diâmetro, com interrupções em pelo menos duas classes intermediárias.

As espécies definidas como secundárias tardias, possuidoras de populações numerosas, e.g., P. heptaphyllum e T. palli$d a$ (Tab. 1), e pouco numerosas, e.g., Esenbeckia grandiflora Mart., Metrodorea nigra A. St.-Hil. e Myroxylon peruiferum L. f. (Tab. 3), apresentaram em comum a ocorrência de indivíduos de pequeno porte, e alturas médias variando entre pouco mais de 2,0 m e 4,8 m, embora possuíssem indivíduos de grande porte. É possível que essas populações tenham se estabelecido recentemente na floresta estudada, especialmente as pouco numerosas, que apresentaram indivíduos que não ultrapassaram $4 \mathrm{~m}$ para altura máxima, e.g., Cestrum sendtnerianum Mart. e Hymenaea courbaril L.

A ausência de indivíduos nas maiores classes diamétricas de C. langsdorffii, pode ser explicada pela retirada de madeira, que pode ser utilizada para práticas de carpintaria (Lorenzi 1992). Foi registrada ainda a ocorrência de outras espécies vegetais possuidoras de madeiras igualmente apreciadas, como Cariniana estrellensis (Raddi) Kuntze (jequitá-branco), Centrolobium tomentosum Guillemin ex Benth. (araribá) e Myroxylon peruiferum L. f. (cabreúva) e de algumas espécies pertencentes ao gênero Ocotea Aubl. (Pinheiro et al. 2002; Pinheiro \& Monteiro 2008). Contudo, não foram encontrados sinais de retirada de madeira, como foi observado por Cavassan et al. (1984), em pontos isolados da floresta estacional estudada por esses autores na Reserva Estadual de Bauru.

\section{Conclusões}

A ocorrência de espécies arbóreas savânicas no interior da floresta estacional semidecidual do JBMB indica a influência da savana florestada contígua, no mecanismo de sucessão secundária florestal. É provável que a savana florestada, pela proximidade, tenha fornecido elementos florísticos logo após as freqüentes queimadas na borda e incêndios ocasionais no interior da floresta estacional (Pimm 1993; Oliveira 1994). Todavia, essas espécies savânicas, através de sementes recém dispersadas ou componentes do banco de sementes, tiveram de dividir, provavelmente, a ocupação inicial de clareiras, na floresta estacional estudada, com 
Tabela 3. Número de indivíduos e valores para altura de espécies secundárias tardias, com populações menores que 10 indivíduos, amostradas na floresta estacional do Jardim Botânico de Bauru. Ni - número de indivíduos amostrados por Pinheiro et al. (2002). Alturas: mínimas (Min.); médias (Med.); máximas (Max.).

\begin{tabular}{|c|c|c|c|c|}
\hline \multirow{2}{*}{ Espécies } & \multirow{2}{*}{$\mathrm{Ni}$} & \multicolumn{3}{|c|}{ Alturas (m) } \\
\hline & & Min. & Med. & Max. \\
\hline Cordia sellowiana Cham. & 5 & 2,0 & 4,0 & 8,0 \\
\hline Faramea latifolia (Cham. \& Schltdl.) DC. & 6 & 1,5 & 2,7 & 5,0 \\
\hline Cestrum sendtnerianum Mart. & 5 & 1,5 & 1,8 & 2,0 \\
\hline Metrodorea nigra A. St.-Hil. & 6 & 1,5 & 3,1 & 5,0 \\
\hline Myroxylon peruiferum L. f. & 6 & 2,0 & 4,8 & 13,0 \\
\hline Psychotria carthagenensis Jacq. & 5 & 2,0 & 3,6 & 5,0 \\
\hline Maytenus floribunda Reiss. & 5 & 1,5 & 2,3 & 5,0 \\
\hline Esenbeckia grandiflora Mart. & 4 & 1,5 & 1,8 & 2,0 \\
\hline Hymenaea courbaril L. & 4 & 2,0 & 3,0 & 4,0 \\
\hline
\end{tabular}

espécies arbustivo-arbóreas florestais, também definidas como iniciais. Essas espécies florestais estariam presentes em clareiras, através de suas sementes ou de plântulas, além de indivíduos jovens, como descreveu Connell (1989).

É muito provável, que a savana florestada tenha atuado como uma zona tampão, nas áreas de contato ao redor da fitocenose florestal, atenuando o efeito de borda (Schelhas \& Greenberg 1996), além de fornecer conexão entre fragmentos florestais, inclusive com fragmento contíguos ao JBMB, facilitando o deslocamento da fauna (Beeby 1993). A proximidade desses fragmentos florestais contíguos representa uma importante condição para a renovação das populações da floresta estacional mantidas pelo jardim botânico municipal. Portanto, a conservação do conjunto de fragmentos florestais e savânicos do entorno do JBMB, é uma medida necessária para a permanência dessas fitocenoses a longo prazo.

\section{Agradecimentos}

Aos doutores Oswaldo Cesar, Osmar Cavassan, Ricardo Ribeiro Rodrigues e aos revisores pelas sugestões que muito ajudaram no aprimoramento deste trabalho. Ao Conselho Nacional de Desenvolvimento Científico $(\mathrm{CNPq})$ e ao programa de pós-graduação em Biologia Vegetal da Universidade Estadual de Campinas (Unicamp) pela bolsa de mestrado concedida. À Secretaria Municipal Ambiente (SEMMA) e ao Jardim Botânico Municipal de Bauru (JBMB) pelo apoio logístico.

\section{Referências bibliográficas}

Barbosa, A.R.; Yamamoto, K. \& Valio, I.F.M. 1999. Effect of light and temperatura on germination and early growth of Vochysia tucanorum Mart., Vochysiaceae, in cerrado and forest soil under different radiation levels. Revista Brasileira de Botânica 22(2): 275-280.

Barnes, B.V.; Zak, D.R.; Denton, S.R. \& Spurr, S.H. 1997. Forest ecology. New York, John Wiley \& Sons.

Bartha, S.; Meiners, S.J.; Pickett, S.T.A. \& Cadenasso, M.L. 2003. Plant colonization windows in a mesic old field succession. Applied Vegetation Science 6: 205-212.

Bazzaz, F. A. \& Pickett, S. T. A. 1980. Physiological ecology of tropical succession: a comparative review. Annual Review of Ecology and Systematics. 11: 287-310.

Beeby, A. 1993. Applying ecology. London, Chapman \& Hall.

Braun-Blanquet, J. 1979. Fitosociologia: bases para el estúdio de las comunidades vegetais. Madrid, $H$. Blume Ediciones.
Brokaw, N.V.L. 1985a. Gap-phase regeneration in a tropical forest. Ecology 66(3): 682-687.

Brokaw, N.V.L. 1985b. Treefalls, regrowth, and community structure in tropical forests. Pp. 53-69. In: S.T.A. Pickett \& P.S. White (eds.). The ecology of natural disturbance and path dynamics. San Diego, Academic Press.

Brokaw, N.V.L. 1989. Species composition in gaps and structure of a tropical forest. Ecology 70(3): 538-541.

Brokaw, N.V.L. \& Scheiner, S.M. 1989. Species composition in gaps and structure of a tropical forest. Ecology 70(3): 538-541.

Cavassan, O.; Cesar, O. \& Martins, F.R. 1984. Fitossociologia da vegetação arbórea da Reserva Estadual de Bauru, estado de São Paulo. Revista Brasileira de Botânica 7(2): 91-106.

Condit, R.; Sukumar, R.; Hubbell, S.P. \& Foster, R.B. 1998. Predicting population trends from size distribuitions: a direct test in a tropical tree community. The American Naturalist 152(4): 495-509.

Connell, J.H. 1989. Some processes affecting the species composition in forest gaps. Ecology 70(3): 560-562.

Durigan, G.; Baitello, J.B.; Franco, G.A.D.C. \& Siqueira, M.F. 2004. Plantas do cerrado paulista: imagens de um paisagem ameaçada. São Paulo, Páginas \& Letras Editora e Gráfica.

Durigan, G. \& Leitão Filho, H.F. 1995. Florística e fitossociologia de matas ciliares do Oeste Paulista. Revista do Instituto Florestal 7(2): 197-239.

Durigan, G. \& Nogueira, J.C.B. 1990. Recomposição de matas ciliares: orientações básicas. São Paulo, Instituto Florestal. (Série Registros, n. 4).

Gandolfi, S.; Leitão Filho, H.F. \& Bezerra, C.L. 1995. Levantamento florístico e caráter sucessional das espécies arbustivo-arbóreas de uma floresta mesófila semidecídua no município de Guarulhos, SP. Revista Brasileira de Biologia 55(4): 753-767.

Gomes, E.P.C.; Fisch, S.T.V. \& Mantovani, W. 2005. Estrutura e composição do componente arbóreo na Reserva Ecológica do Trabiju, Pindamonhangaba, SP, Brasil. Acta Botanica Brasílica 19(3): 451-464.

Grime, J.P. \& Hillier, S.H. 1993. The contribution of seedling regeneration to the structure and dynamics of plant. Communities and larger units of landscape. Pp. 349-364. In: M. Fenner (ed.). Seeds. The ecology of regeneration in plant communities. Wallingford, $\mathrm{CAB}$ International.

Hatfield, J.S.; Link, W.A. \& Dawson, D.K. 1996. Coexistence and community structure of tropical trees in a Hawaiian montane rain forest. Biotropica 28(4b): 746-758.

Higgins, S.I.; Pickett, S.T.A. \& Bond., W.J. 2000. Predicting extinction risks for plants: environmental stochasticity can save declining populations. Trends of Ecology and Evolution 15(12): 516-520.

Leitão Filho, H.F. 1992. A flora arbórea dos cerrados do Estado de São Paulo. Hoehnea 19(1/2): 151-163.

Lobo, P.C. \& Joly, C.A. 2000. Aspectos ecofisiológicos da vegetação de mata ciliar do Sudeste do Brasil. P.p. 143-157. In: R.R. Rodrigues \& H.F. Leitão filho (eds.). Matas ciliares: conservação e recuperação. São Paulo, Editora da Universidade de São Paulo, Fapesp. 
Longman, K.A. \& Jeník, J. 1992. Forest-savanna boundaries: general considerations. Pp. 3-20. In: P.A. Furley, J. Proctor, J.A. Ratter (eds.). Nature and dynamics of forest-savanna boundaries. London, Chapman \& Hall.

Lopes, W.P.; Paula, A.; Sevilha, A.C. \& Silva, A.F. 2002a. Composição da flora arbórea de um trecho de floresta estacional no jardim botânico da Universidade Federal de Viçosa (face sudoeste), Viçosa, Minas Gerais. Revista Árvore 26(3): 339-347.

Lopes,W.P.; Silva, A.F.; Souza, A.L. \& Meira Neto, J.A.A. 2002b. Estrutura fitossociológica de um trecho de vegetação arbórea no Parque Estadual do Rio Doce - Minas Gerais, Brasil. Acta Botanica Brasilica 16(4): 443-456.

Lorenzi, H. 1992. Árvores brasileiras: manual de identificação e cultivo de plantas arbóreas nativas do Brasil. Nova Odessa, Plantarum.

Lorenzi, H. 2000. Plantas daninhas do Brasil: terrestres, aquáticas, parasitas e tóxicas. Nova Odessa, Instituto Plantarum.

Lorenzi, H. 2002. Árvores brasileiras: manual de identificação e cultivo de plantas arbóreas nativas do Brasil. Nova Odessa, Plantarum.

Lorenzi, H. \& Souza, H.M. 2001. Plantas ornamentais no Brasil: arbustivas, herbáceas e trepadeiras. Nova Odessa, Instituto Plantarum.

Marangon, L.C.; Soares, J.J.; Feliciano, A.L.P.; Lins, C.F. \& Brandão, S. 2007. Estrutura fitossociológica e classificação sucessional do componente arbóreo de um fragmento de floresta estacional semidecidual, no município de viçosa, minas gerais. Cerne. 13(2): 208-221.

Martens, S.N.; Breshears, D.D. \& Meyer, C.W. 2000. Spatial distributions of understory light along the grassland/forest continuum: effects of cover, height, and spatial patterns of tree canopies. Ecological Modelling 126: 79-93.

Martins, F.R. 1991. Estrutura de uma floresta mesófila. Campinas, Editora da UNICAMP.

Mesquita, R.C.G.; Ickes, K.; Ganade, G. \& Williamson, G.B. 2001. Alternative successional pathways in the Amazon basin. The Journal of Ecology 89(4): 528-537.

Mueller-Dombois, D. 1995. Biological diversity and disturbance regimes in island ecosystems. Pp. 163-175. In: P.M. Vitousek, L.L. Loope \& H. Adsersen (eds.). Islands: biological diversity and ecosystem function. Berlin, Springer-Verlag.

Nascimento, A.R.T.; Felfili, J.M. \& Meirelles, E.M. 2004. Florística e estrutura da comunidade arbórea de um remanescente de Floresta Estacional Decidual de encosta, Monte Alegre, GO, Brasil. Acta Botanica Brasilica 18(3): 659-669.

Newman, E.I. 1982. Niche separation and species diversity in terrestrial vegetation. Pp. 61-77. In: E.I. Newman (ed.). The plant community as a working mechanism. Oxford, Blackwell Scientific Publications.

Nunes, Y.R.F.; Mendonça, A.V.R.; Botezelli, L.; Machado, E.L.M. \& OliveiraFilho, A.T. 2003. Variações da fisionomia, diversidade e composição de guildas da comunidade arbórea em um fragmento de floresta semidecidual em Lavras, MG. Acta Botanica Brasilica 17(2): 213-229.

Oliveira, P. E. 1994. Aspectos da reprodução de plantas de cerrado e conservação. Boletim do Herbário Ezequias Heringer 1: 34-45.

Oliveira-Filho, A.T. \& Ratter, J.A. 2002. Vegetation physiognomies and wood flora of the cerrado biome. P.p. 91-119. In: P.S. Oliviera \& R.J. Marquis (eds.). The cerrados of Brazil: ecology and natural history of a neotropical savanna. New York, Columbia University Press.

Oliveira-Filho, A.T.; Camisão Neto, A.A. \& Volpato, M.M. 1996. Structure and dispersion of four three populations in an area of montane semideciduous forest in Southeasten Brazil. Biotropica 28(4b): 762-769.

Oliveira-Filho, A.T.; Mello, J.M. \& Scolforo, J.R. 1997. Effects of past disturbance and edges on tree community structure and dynamics within a fragment of tropical semideciduous forest in South-eastern Brazil over a five-year period (1987-1992). Plant Ecology 131: 45-66.

Peixoto, G.L.; Martins, S.V.; Silva, A.F. \& Silva, E. 2005. Estrutura do componente arbóreo de um trecho de Floresta Atlântica na Área de Proteção Ambiental da Serra da Capoeira Grande, Rio de Janeiro, RJ, Brasil. Acta Botanica Brasilica 19(3): 539-547.

Pickett, S.T. \& White, P.S. 1986. The ecology of natural disturbance and patch dynamics. Orlando, Academic Press.

Pickett, S. T.A.; Collins, S. L. \& Armesto, J. J. 1987. A hierarchical consideration of causes and mechanisms of succession. Vegetatio 69: 109-11.
Pimm, S.L. 1993. Biodiversity and the balance of nature. P.p. 347-359. In: E.D. Schulze \& H.A. Mooney (eds.). Biodiversity and ecosystem function. Berlin: Springer-Verlag.

Pinheiro, M.H.O.; Monteiro, R. \& Cesar, O. 2002. Levantamento fitossociológico da floresta estacional semidecidual do Jardim Botânico Municipal de Bauru, São Paulo. Naturalia 27: 145-164.

Pinheiro, M.H.O. \& Monteiro, R. 2006. Contribution of forest species to the floristic composition of a forested savanna in Southeastern Brazil. Brazilian Archives of Biology and Technology 49(5): 763-774.

Pinheiro, M.H.O. \& Monteiro, R. 2008. Florística de uma floresta estacional semidecidual, localizada em ecótono savânico-florestal, no município de Bauru, SP, Brasil. Acta Botanica Brasilica 22(4): 1085-1094.

Pinto, L.V.A.; Botelho, S.A.; Oliveira-Filho, A.T. \& Davide, A.C. . 2005. Estudo da vegetação como subsídios para propostas de recuperação das nascentes da bacia hidrográfica do Ribeirão Santa Cruz, Lavras, MG. Revista Árvore 29(5):775-793.

Remmert, H. 1991. The mosaic-cycle concept of ecosystems - an overview. Pp. 1-21. In: H. Remmert, (ed.). The mosaic-cycle concept of ecosystems. Berlin, Springer-Verlag.

Rodrigues, L.A.; Carvalho, D.A.; Oliveira-Filho, A.T.; Botrel, R.T. \& Silva, E.A. 2003. Florística e estrutura da comunidade arbórea de um fragmento florestal em Luminárias, MG. Acta Botanica Brasilica 17(1): 71-87.

Rodrigues, R.R. 2000. Uma discussão nomenclatural das formações ciliares. Pp. 91-99. In: R.R. Rodrigues \& H.F. Leitão filho (eds.). Matas ciliares: conservação e recuperação. São Paulo, Editora da Universidade de São Paulo, Fapesp.

Saunders, D.A.; Hobbs, R.J. \& Margules, C.R. 1991. Biological consequences of ecosystem fragmentation: a review. Conservation Biology 5(1): 18-32.

Schelhas, J. \& Greenberg, R. 1996. Introduction: the value of forest patches. P.p. xv-xxxvi. In: J. SCHELHAS, \& R. GREENBERG (eds.). Forest patches in tropical landscapes. Washington DC: Island Press.

Schupp, E.W.; Howe, H.F.; Augspurger, C.K. \& Levey, D.J. 1988. Arrival and survival in tropical treefall gaps. Ecology 70(3): 562-564.

Souza, D.R.; Souza, A.L.; Leite, H.G. \& Yared, J.A.G. 2006. Análise estrutural em floresta ombrófila densa de terra firme não explorada, Amazônia Oriental. Revista Árvore 30(1): 75-87.

Spiegel, M.R. 1976. Estatística. São Paulo, Editora McGraw-Hill do Brasil.

Stranghetti, V.; Ituralde, R.B.; Gimenez, L.R.\& Almella, D. 2003. Florística de um fragmento florestal do sítio São Pedro, município de Potirendaba, Estado de São Paulo. Acta Scientiarum. 25(1): 167-172.

Tabanez, A.A.J.; Viana, V.M. \& Dias, A.S. 1997. Conseqüências da fragmentação e do efeito de borda sobre a estrutura, diversidade e sustentabilidade de um fragmento de floresta de planalto de Piracicaba, SP. Revista Brasileira de Biologia 57(1): 47-60.

Tabarelli, M. \& Mantovani, W. 1997a. Ocupação de clareiras naturais na floresta na Serra da Cantareira. Naturalia 22: 89-102.

Tabarelli, M. \& Mantovani, W. 1997b. Colonização de clareiras na floresta atlântica no sudeste do Brasil. Revista Brasileira de Botânica 20(1): 57-66.

Terborgh, J. 1992. Maintenance of diversity in tropical forests. Biotropica 24(2b): 283-292.

Terborgh, J.; Flores, C.; Mueller, P. \& Davenport, L. 1997. Estimating the ages of successional stands of tropical trees from growth increments. Journal of Tropical Ecology 14: 833-836.

Veloso, H.P. 1992. Sistema fitogeográfico. Pp. 9-38. In: Manual técnico da vegetação brasileira (Manuais técnicos em geociências, 1). Rio de Janeiro, Fundação Instituto Brasileiro de Geografia e Estatística (IBGE).

Viana, V.M. \& Tabanez, A.A.J. 1996. Biology and conservation of forest fragments in the Brazilian Atlantic moist forest. Pp. 151-167. In: J. Schelhas, R. Greenberg (eds.). Forest patches in tropical landscapes. Washington DC, Island Press.

Whitmore, T.C. 1982. On pattern and process in forests. Pp. 45-59. In: E.I. Newman (ed.). The plant community as a working mechanism. Oxford, Blackwell Scientific Publications.

Whitmore, T.C. 1989. Canopy gaps and the major groups of forest trees. Ecology 70(3): 536-538.

Versão eletrônica do artigo em www.scielo.br/abb e http://www.botanica.org.br/acta/ojs 